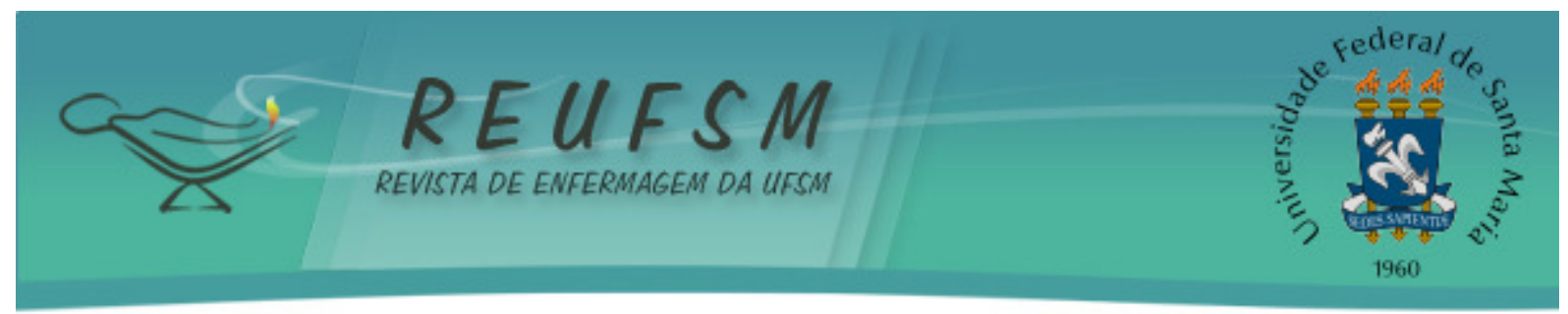

ARTIGO ORIGINAL

\title{
INTERNAÇÕES POR QUEDAS NO RIO GRANDE DO SUL: INTERVENÇÕES DE ENFERMAGEM PARTINDO DE FATORES AMBIENTAIS
}

\author{
HOSPITALIZATIONS DUE TO FALLS IN RIO GRANDE DO SUL: INTERVENTIONS FOR \\ NURSING CONCERNING ENVIRONMENTAL FACTORS
} HOSPITALIZACIONES POR CAÍDAS EN RIO GRANDE DO SUL: INTERVENCIONES DE
ENFERMERÍA PARTIENDO DE FACTORES AMBIENTALES

Maíra Rossetto

Doi: $10.5902 / 2179769213641$

Marta Julia Marques Lopes ${ }^{3}$

RESUMO: Objetivo: explorar registros de quedas no Rio Grande do Sul, relacionando esses
com potenciais de intervenção da enfermagem para a redução desses eventos. Método:
trata-se de um estudo ecológico descritivo, que apresenta os dados de internações por
quedas entre os anos de 2008 a 2012 , acessados no Departamento de Informática do
Sistema Único de Saúde. Resultados: as quedas do mesmo nível por escorregão, tropeção
ou passos em falso perfizeram $63,1 \%$ dos registros no período do estudo. Encontraram-se
indicativos nas taxas de mortalidade hospitalar e na média de permanência hospitalar que
sugerem, referência da demanda de maior complexidade para os serviços de saúde.
Conclusão: a implementação de medidas ambientais como redução de tapetes e degraus,
adoção de material emborrachado no piso, barras de apoio, camas com guardas de
proteção e iluminação sensivel ao movimento, associados a medidas educativas poderia
reduzir o número de internações com quedas. Descritores: Enfermagem; Acidentes por quedas; Riscos ambientais; Sistemas de informação.

ABSTRACT: Objective: to explore records of falls in Rio Grande do Sul, relating these to potential nursing intervention to reduce these events. Method: this is a descriptive ecological study, which presents data on hospitalizations for falls between the years 2008 to 2012, accessed at the Department of the Unified Health System. Results: falls at the same level caused by slipping, tripping or missteps amounted to $63.1 \%$ of the records during the study period. The indications found in hospital mortality rates and mean hospital stay suggest reference to more complex demands for health services. Conclusion: the implementation of environmental measures such as reduction of carpets and stairs, adoption of rubberized material on the floor, grab bars, beds with protective guards and sensitive to motion lighting, combined with educational measures could reduce the number of hospitalizations with falls.

Descriptors: Nursing; Accidental falls; Environmental risks; Information systems.

RESUMEN: Objetivo: explorar los registros de caídas en Rio Grande do Sul, relacionándolos con las potenciales intervenciones de enfermería para reducir estos eventos. Metodo: estudio ecológico descriptivo, que presenta datos sobre las hospitalizaciones por caídas

\footnotetext{
${ }^{1}$ Enfermeira. Mestra. Doutoranda em Enfermagem pela Universidade Federal do Rio Grande do Sul (UFRGS). Porto Alegre, RS, Brasil. E-mail: maira_rossetto@hotmail.com

2 Enfermeiro. Mestre. Doutorando em Enfermagem pela Universidade Federal do Rio Grande do Sul (UFRGS). Porto Alegre, RS, Brasil. E-mail: almachadobueno@gmail.com

${ }^{3}$ Enfermeira. Doutora. Professora na Escola de Enfermagem da Universidade Federal do Rio Grande do Sul (UFRGS). Porto Alegre, RS, Brasil. E-mail: malopes@gmail.com
} 


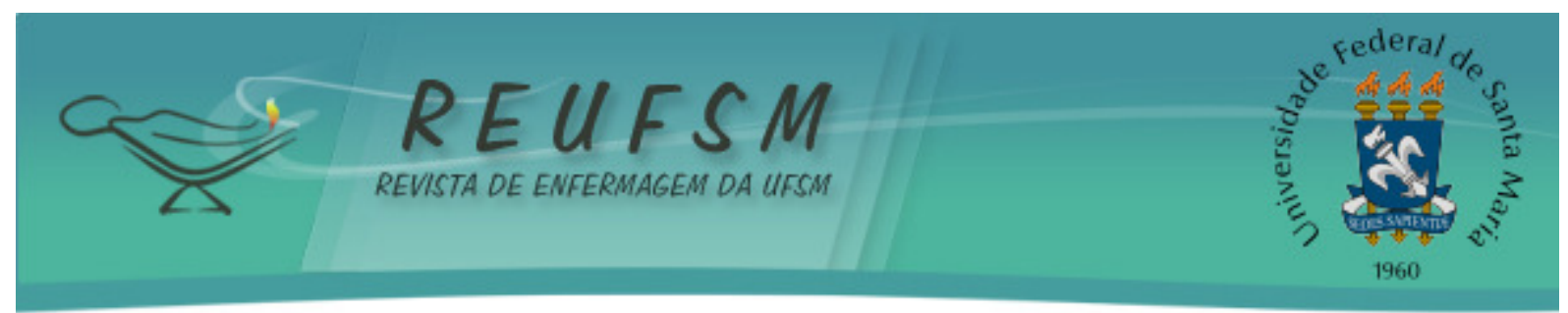

entre los años 2008 a 2012, consultados en el Departamento de Informática del Sistema Único de Salud. Resultados: las caídas del mismo nivel por resbalones, tropezones o traspiés ascendió al $63.1 \%$ de los registros durante el período de estudio. Hemos encontrado indicios en las tasas de mortalidad hospitalaria y la estancia hospitalaria media, referencia a la demanda de servicios de salud de mayor complejidad. Reflexiones finales: la aplicación de medidas medioambientales como la reducción de alfombras y escalones, la adopción de material de goma en el piso, barras de apoyo, camas con guardas de protección o iluminación sensible al movimiento, asociadas a medidas educativas podría reducir el número de hospitalizaciones por caídas.

Descriptores: Enfermería; Accidentes por caídas; Riesgos ambientales; Sistemas de información.

\section{INTRODUÇÃO}

As quedas podem ser definidas como eventos não intencionais resultantes do deslocamento do corpo para um nível inferior à posição inicial, provocado por circunstâncias multifatoriais, resultando ou não em dano. ${ }^{1}$ Tais eventos colocam-se com uma das prioridades das agendas públicas de saúde devido às graves consequências físicas e psicológicas que acarretam as vítimas e familiares, além dos altos custos governamentais com tratamentos e recuperação. ${ }^{2}$

A amplitude desta problemática pode ser verificada por meio dos dados do Sistema de Informações Hospitalares (SIH) do Sistema Único de Saúde (SUS) que, para os anos de 2008 a 2012 contabilizaram 1.726.427 internações por quedas no Brasil. No mesmo ano, a taxa de mortalidade hospitalar foi de 1,88 para cada 100 internações, com gasto médio de $R \$ 843,71$ e 4,6 dias, em média, de permanência hospitalar. ${ }^{3}$

Nesse contexto, para os mesmos anos somaram-se 115.834 autorizações de internação Hospitalar (AlH) por quedas no Rio Grande do Sul (RS), colocando o estado na quarta posição entre as unidades da federação em número de $\mathrm{AlH} .{ }^{4} \mathrm{O}$ RS ocupa o sétimo lugar no que se refere aos gastos com tratamentos das quedas com valores de R\$ 886,53 por AlH. A média de permanência hospitalar é a décima terceira do país e a taxa de mortalidade hospitalar ocupa a quinta posição com 1,9 óbitos por 100 internações. ${ }^{4}$ Esses dados são expoentes de uma condição epidemiológica que situa as quedas como um problema de saúde pública evitável, sendo, muitas vezes, o causador de danos irreversíveis que podem impactar na qualidade de vida das pessoas.

Alguns fatores intrínsecos e extrínsecos são determinantes de situações predisponentes as quedas. Os fatores intrínsecos são relacionados a própria pessoa, como por exemplo idade, doenças crônicas, uso de fármacos, depressão, diminuição da cognição, redução da capacidade funcional, entre outras. Enquanto os fatores extrínsecos estão relacionados com o meio ambiente e, também, podem influenciar a ocorrência de quedas. ${ }^{5}$

Esse panorama indica que, sendo passíveis de prevenção, as quedas necessitam de intervenções educativas dos profissionais da saúde, visando à alteração de atitudes, à adequação de ambientes e a redução dos danos gerados por estes acidentes. Considera-se que os profissionais de enfermagem, por exemplo, por terem como prática essencial o cuidado, podem, especialmente, contribuir na redução das taxas de internações por quedas, atuando, por meio do conhecimento prévio dos fatores ambientais, na prevenção desse tipo de evento. Nessa perspectiva, entende-se que o entendimento dos fatores ambientais envolvidos em situações de quedas pode subsidiar a educação em saúde e a implementação, local e descentralizada, de medidas preventivas adaptadas as diferentes realidades constituinte desses agravos. ${ }^{1,6}$

Nesse sentido, a abordagem pretendida neste estudo relaciona-se aos fatores ambientais predisponentes as quedas na sua origem, ou seja, pretende-se discutir as quedas do ponto de vista preventivo, como evento capaz de ser evitado. Assim, ao discutir 


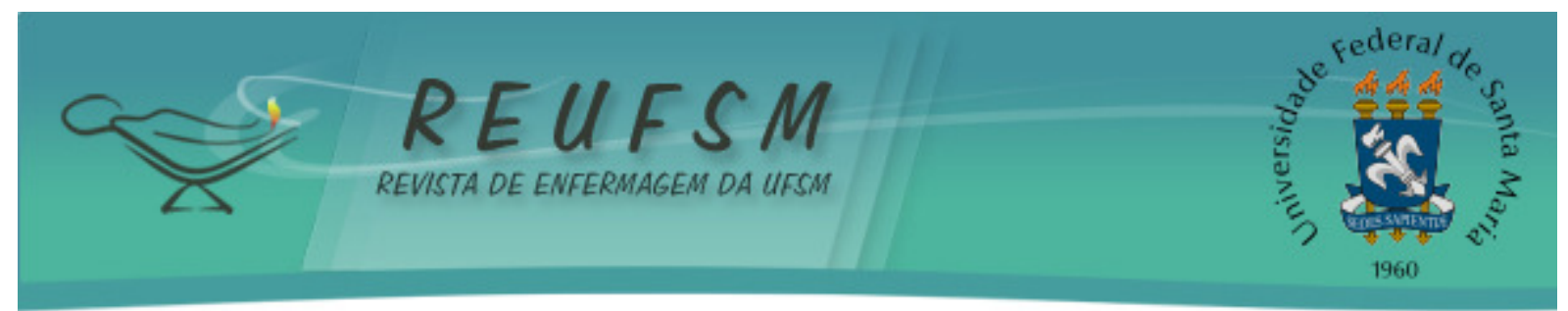

essa problemática e a influência de alguns fatores ambientais nessa casuística, coleta-se subsídios no Sistema de Informática do SUS (DATASUS), a partir dos dados de internação, para melhor compreender este fenômeno, auxiliando, assim, no planejamento do cuidado em enfermagem em seus diferentes âmbitos de atuação. ${ }^{2,5,7}$

A partir do exposto este estudo teve como objetivo explorar os registros de quedas no RS, entre os anos de 2008 a 2012, com base no DATASUS, relacionando esses dados com potenciais de intervenção da enfermagem para a redução desses eventos. Salienta-se que as categorias selecionadas, apresentam-se como fatores ambientais relacionados às internações por quedas no domicílio.

\section{MÉTODO}

Trata-se de um estudo ecológico ${ }^{8}$ descritivo, que apresenta os dados de morbidade (internações) por quedas entre os anos de 2008 a 2012 no RS, registrados nas subcategorias W01- Queda no mesmo nível por escorregão, tropeção ou passos em falsos, W06- Queda de um leito, W07- Queda de uma cadeira, W08- Queda de outro tipo de mobília, W10-Queda em ou de escadas ou degraus da Classificação Internacional de Doenças (CID-10). Essas subcategorias foram selecionadas para discutir a dimensão dos fatores ambientais que podem contribuir para a ocorrência de quedas no domicílio e que podem ser passíveis de intervenção preventiva.

Os dados foram acessados no DATASUS ${ }^{3}$ e selecionados online via Tabnet, segundo - local de internação. As variáveis utilizadas no estudo, excluídos os dados ignorados, foram: sexo, faixa etária, raça/cor, Autorizações de Internação Hospitalar (AlH) aprovadas, caráter de atendimento, regime (tipo de vínculo que a Unidade Hospitalar tem com o SUS), taxa de mortalidade (razão entre a quantidade de óbitos e o número de $\mathrm{AlH}$ aprovadas, computadas como internações, no período, multiplicada por 100), média de permanência hospitalar em dias (média de permanência das internações referentes às AlH aprovadas computadas como internações no período), o número de Autorizações de Internação Hospitalar $(\mathrm{AlH})$ e o valor médio das $\mathrm{AlH}$ aprovadas no período. A busca dos dados foi realizada nos meses de outubro e novembro de 2013, no site do Ministério da Saúde, com posterior compilação e análise dos dados coletados.

Aplicaram-se às variáveis o Coeficiente de Correlação de Person a fim de verificar o grau de associação linear entre algumas variáveis. Para verificar a normalidade na distribuição das variáveis utilizou-se o teste de Shapiro-Wilk com intervalo de confiança de 95\%. A análise dos dados foi realizada com base no programa TabWin e com auxílio de uma planilha eletrônica. Os dados foram coletados no DATASUS seguindo um roteiro estabelecido pelos autores. O projeto não passou por avaliação em comitê de ética em pesquisa, pois o banco de dados utilizado é de domínio público.

\section{RESULTADOS E DISCUSSÃO}

A análise dos dados a seguir apresenta 8.280 registros de internações por quedas, correspondentes ao período de 2008 a 2012 no estado do RS. As internações por quedas do mesmo nível com origem em escorregões, tropeção ou passos em falso foram responsáveis por $63,1 \%$ dos registros. As quedas relativas às cadeiras, escadas ou degraus, juntas, perfizeram $31,7 \%$ dos dados, sendo às quedas do leito e de outros tipos de mobília $5,2 \%$ das internações no período (Tabela 1). 


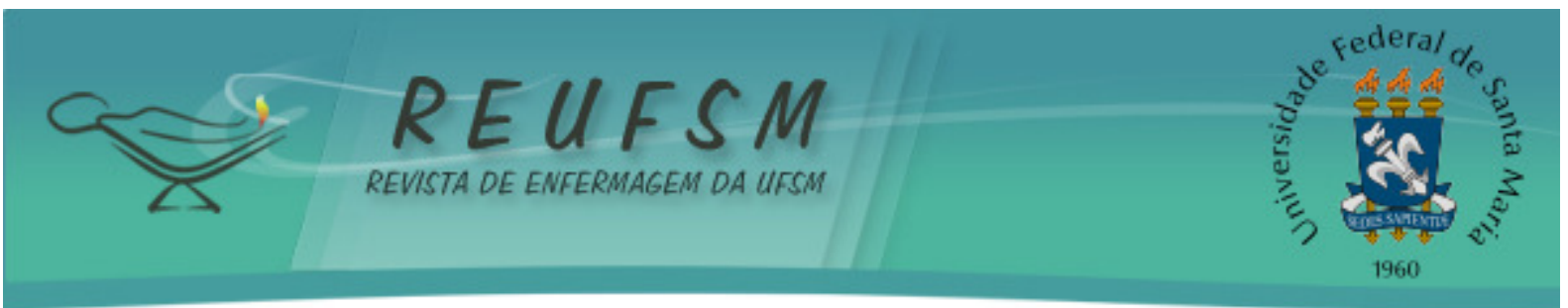

Tabela 1 - Internações por quedas, no período de 2008 a 2012, no Rio Grande do Sul.

\begin{tabular}{cccccccccccccc}
\hline \multirow{2}{*}{ Categorias* $^{2}$} & \multicolumn{2}{c}{2008} & \multicolumn{2}{c}{2009} & \multicolumn{2}{c}{2010} & \multicolumn{2}{c}{2011} & \multicolumn{2}{c}{2012} & \multicolumn{3}{c}{ Total } \\
\cline { 2 - 14 } & $\mathbf{N}$ & $\%$ & $\mathbf{n}$ & $\%$ & $\mathbf{N}$ & $\%$ & $\mathbf{n}$ & $\%$ & $\mathbf{n}$ & $\%$ & $\mathbf{n}$ & $\%$ \\
\hline W01 & 518 & 9,9 & 867 & 16,6 & 1319 & 25,2 & 1319 & 25,2 & 1201 & 23,0 & 5224 & 100 \\
W06 & 115 & 39,8 & 38 & 13,1 & 44 & 15,2 & 45 & 15,6 & 47 & 16,3 & 289 & 100 \\
W07 & 1135 & 82,9 & 100 & 7,3 & 64 & 4,7 & 50 & 3,7 & 20 & 1,5 & 1369 & 100 \\
W08 & 17 & 12,2 & 20 & 14,4 & 36 & 25,9 & 43 & 30,9 & 23 & 16,5 & 139 & 100 \\
W10 & 345 & 27,4 & 243 & 19,3 & 330 & 26,2 & 219 & 17,4 & 122 & 9,7 & 1259 & 100 \\
\hline
\end{tabular}

Fonte: Ministério da Saúde - Sistema de Informações Hospitalares do SUS (SIH/SUS)

*Categorias CID-10: W01 - Queda no mesmo nível por escorregão, tropeção ou passos em falsos, W06 - Queda de um leito, W07 - Queda de uma cadeira, W08 - Queda de outro tipo de mobília e W10 Queda em ou de escadas ou degraus.

A taxa de internações por quedas, para 10.000 habitantes, no período do estudo indica uma tendência à redução das internações nos últimos três anos (Figura 1). Contudo, verifica-se que essa taxa oscilou ao longo do período, com maior taxa em $2008(2,0)$ e a menor $(1,2)$ em 2009.

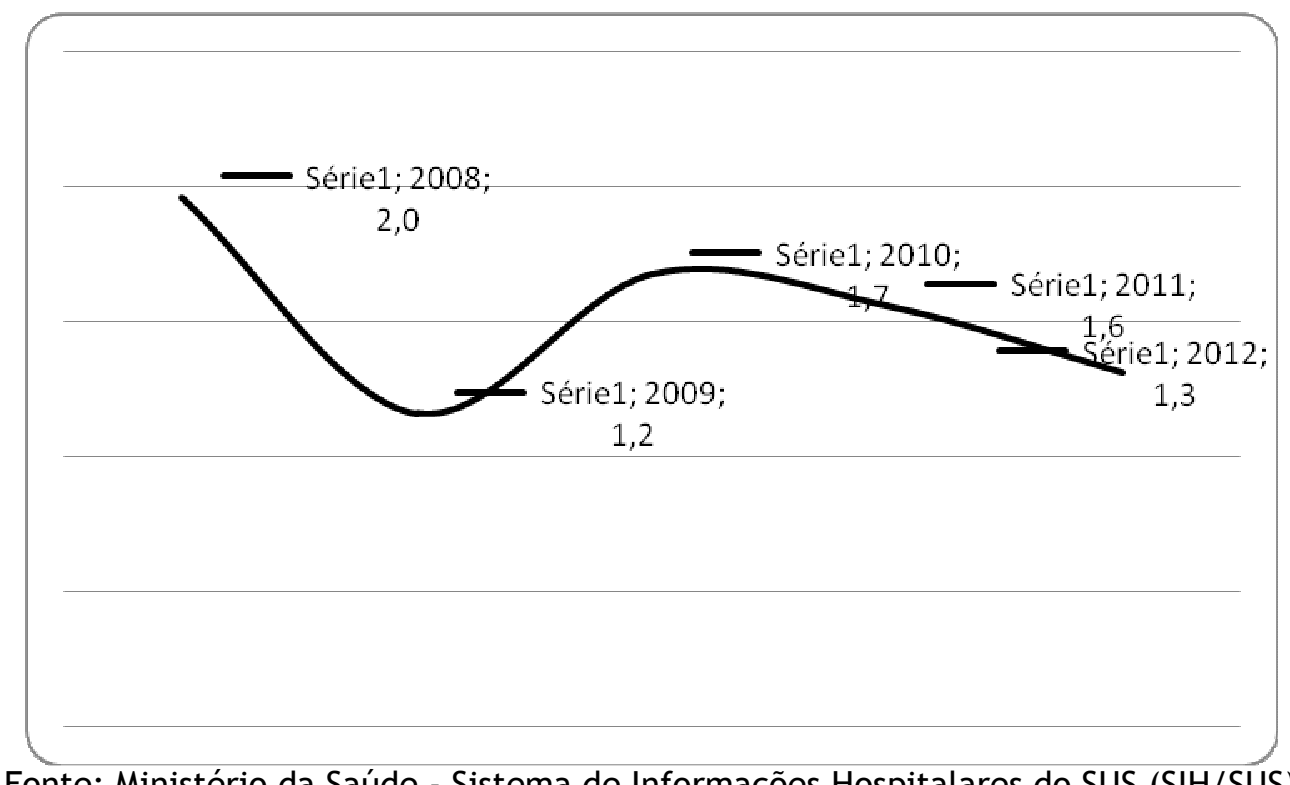

Fonte: Ministério da Saúde - Sistema de Informações Hospitalares do SUS (SIH/SUS)

Figura 1 - Taxa de internações por quedas para 10.000 habitantes.

No que se refere à faixa etária, os indivíduos de 20-59 anos apresentaram 52,5\% do total das taxas de quedas (Tabela 2). Entretanto, para as taxas populacionais ponderadas os idosos (60 anos ou mais) apresentaram, para cada 1.000 habitantes, 2,3 vezes mais internações por quedas do que os indivíduos de 20 a 59 anos, levando-nos a observar uma maior vulnerabilidade de indivíduos idosos para a ocorrência de quedas.

Existem projeções para o ano de 2025 de que o número de indivíduos com idade igual ou superior a 60 anos poderá ser de 32 milhões de habitantes, mostrando a relevância de estudos que viabilizem a construção de políticas públicas de saúde capazes de interferir, positivamente, na diminuição desses agravos. ${ }^{9}$ Essas projeções apontam para um impacto considerável na concepção das práticas profissionais nos serviços de saúde e para a rede de atenção de forma de geral. 


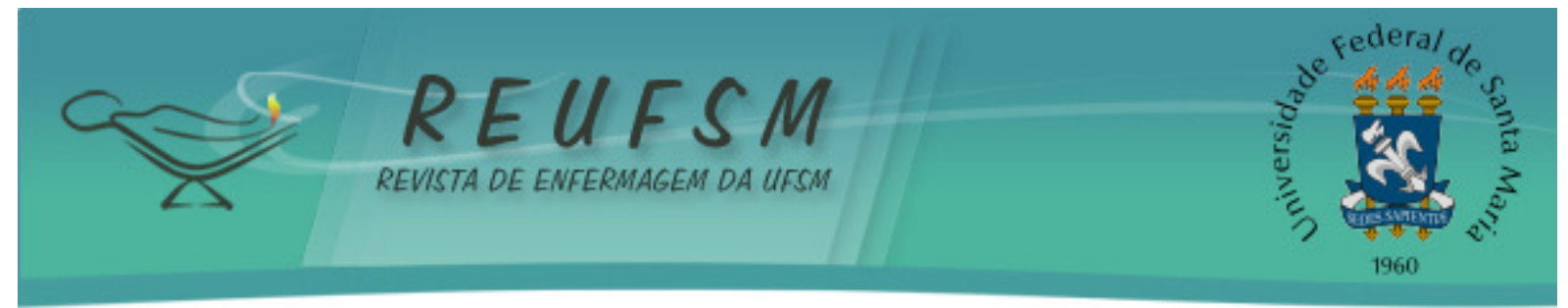

Tabela 2 - Internações por quedas segundo sexo, faixa etária e raça/cor.

\begin{tabular}{lll}
\hline Variável & N & $\%$ \\
\hline $\begin{array}{l}\text { Sexo } \\
\text { Masculino }\end{array}$ & 4.976 & 60,1 \\
$\quad$ Feminino & 3.304 & 39,9 \\
Faixa etária & & \\
Menor de1 ano & 97 & 1,2 \\
1 a 9 anos & 679 & 8,2 \\
10 a 19 anos & 810 & 9,8 \\
20 a 59 anos & 4.348 & 52,5 \\
60 anos ou mais & 2.346 & 28,3 \\
Raça/Cor & & \\
Branca & 6.213 & 92,2 \\
Preta & 293 & 4,3 \\
Parda & 211 & 3,1 \\
Amarela & 17 & 0,3 \\
Indígena & 3 & 0,01 \\
\hline
\end{tabular}

Fonte: Ministério da Saúde - Sistema de Informações Hospitalares do SUS (SIH/SUS)

CategoriasCID-10: W01 - Queda no mesmo nível por escorregão, tropeção ou passos em falsos, W06 Queda de um leito, W07 - Queda de uma cadeira, W08 - Queda de outro tipo de mobília e W10 Queda em ou de escadas ou degraus.

Esses dados evidenciam situações de vulnerabilidade geracional e sugerem a população idosa como a mais vulnerável para a ocorrência das quedas, pois, muitas vezes, os idosos apresentam sua capacidade funcional reduzida. Em ambientes com móveis e objetos espalhados pelo chão, tapetes soltos, pouca iluminação e pisos escorregadios, a ocorrência de escorregão ou queda pode ser facilitada. Assim, é na interação desses fatores com espaços vividos e percebidos, ou não, como risco para quedas que se produzem e reproduzem situações favoráveis à ambientes predisponentes a esses eventos. A existência de obstáculos ambientais pode predispor o indivíduo ao acidente por queda, principalmente dentro da própria moradia, seja ela em domicílio próprio ou em instituições de saúde. A queda pode ser considerada um evento sentinela na vida do idoso, marcador potencial do início de importante declínio da função e/ou sintoma de uma doença. ${ }^{6}$

O crescente aumento da população de idosos e algumas mudanças na estrutura familiar, tem levado ao aumento desse grupo de pessoas residindo sozinhos, desenvolvendo as tarefas cotidianas e mesmo o autocuidado em saúde. A partir desses novos rearranjos, o cuidado desses indivíduos, o planejamento e a readequação do ambiente necessitam considerar suas necessidades e peculiaridades para o seu autocuidado. Destaca-se que, a residência é o local mais frequente de ocorrência de causas externas de morbimortalidade (acidentes e violências), as quais determinam atendimentos nas emergências hospitalares, e demonstram a importância dos acidentes domésticos como responsável por parcela considerável dos dados de morbidade hospitalar. ${ }^{10}$

Aos profissionais de saúde envolvidos no cuidado, caberá identificar fatores de riscos domésticos e, a partir destes, desenvolverem ações educativas que diminuam a fragilidade do cotidiano para prevenir as quedas. ${ }^{9}$ Salienta-se que as ações educativas a partir da enfermagem necessitam corresponder as necessidades individuais de cada usuário dos serviços, incumbindo a esse profissional construir as interlocuções entre teoria e prática, com soluções inteligíveis e aplicáveis as diferentes realidades socioeconômicas, ambientais e físicas do processo de cuidar para prevenção de quedas. ${ }^{6}$ 


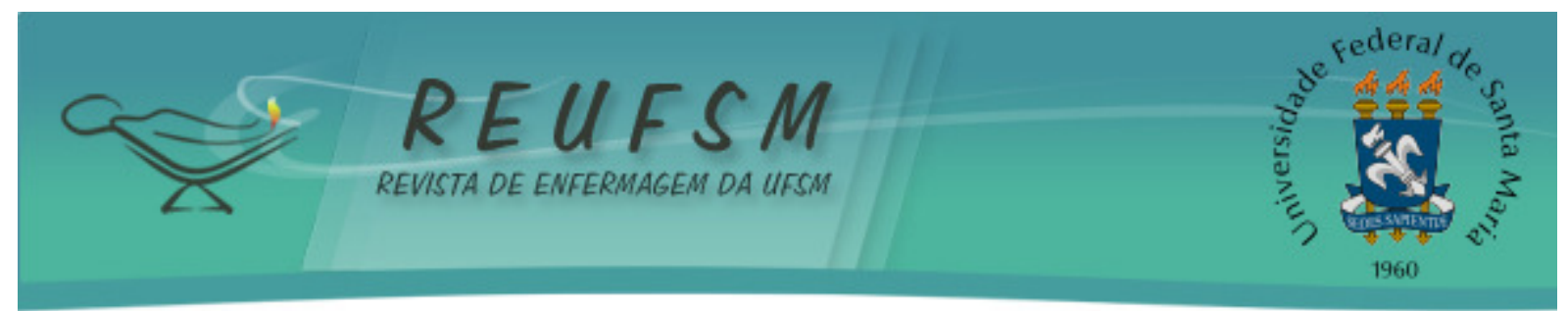

As quedas, em muitos casos, podem ser evitadas por meio de ações de prevenção, no entanto, quando ocorrem, ocasionam consequências sérias como traumas, fraturas, coma e óbito. Nesses casos, a internação hospitalar é acessada para a reabilitação do indivíduo, e produz impactos diretos na sua qualidade de vida. A alta proporção de quedas, responsável por 39,3\% dos atendimentos em unidades de emergência dos hospitais brasileiros, aponta a necessidade de planejar intervenções e novos estudos com vistas à determinação dos principais fatores de risco. ${ }^{10}$

A análise dos dados quanto ao sexo das vítimas indicou que os homens são mais vitimados, tanto para números absolutos quanto para taxas ponderadas da população (Tabela 2). Os homens apresentam taxas ponderadas populacionais de uma internação para cada 1.000 habitante, enquanto as taxas para as mulheres são de 0,6 internações para o mesmo número de habitantes, corroborando com outros estudos. ${ }^{10-11}$

Os dados populacionais ponderados para raça/cor confirmam a tendência dos dados absolutos ao indicarem que os indivíduos brancos são mais vítimas de internações por quedas no período (Tabela 2). Verificou-se que os pessoas brancas ocupam leitos hospitalares por quedas 1,4 vezes mais que os pretos e amarelos, 3,5 mais que os pardos e sete vezes mais que os indígenas.

A Tabela 3 apresenta a relação do caráter e do regime de atendimento com a taxa de mortalidade e a média de permanência. A relação da taxa de mortalidade com o caráter de atendimento, embora não significante do ponto de vista estatístico, sugere que o aumento do número de internações de urgência diminui as taxas de mortalidade e praticamente não altera as taxas de permanência hospitalar.

Nos atendimentos de caráter eletivo, na medida em que ocorre um aumento dessa característica, as médias de permanência hospitalar e as taxas de mortalidade também se elevam, no entanto não houve significância estatística nessa relação. No que se refere ao regime privado (hospitais contratados, filantrópicos, filantrópicos isentos de tributos e contribuições sociais e de pertencentes a sindicatos) o coeficiente de correlação indicou relação inversa e significativa, do ponto de vista estatístico, com a taxa de mortalidade, identificando que os atendimentos em serviços privados contratados pelo SUS atuam como fator protetivo contra mortalidade e a longa permanência hospitalar, embora para os dados relacionados à longa permanência não obtiveram significância na associação proposta. O regime público (hospitais federais, estaduais e municipais) apresentou coeficiente de correlação fraco e estatisticamente insignificante para mortalidade e moderado para permanência hospitalar.

Tabela 3 - Regime e caráter de atendimento nas internações por quedas, no período de 2008 a 2012, no Rio Grande do Sul.

\begin{tabular}{|c|c|c|c|c|}
\hline \multirow{2}{*}{ Variável } & \multirow{2}{*}{$\mathbf{N}$} & \multirow{2}{*}{$\%$} & \multicolumn{2}{|c|}{ Coeficiente de Correlação de Pearson } \\
\hline & & & Taxa de mortalidade & Média de permanência \\
\hline \multicolumn{5}{|c|}{ Caráter de atendimento* } \\
\hline Eletivo & 1.567 & 20,3 & 0,88 & 0,80 \\
\hline Urgência & 6.146 & 79,7 & $-0,11$ & 0,20 \\
\hline \multicolumn{5}{|l|}{ Regime } \\
\hline Público & 2.561 & 30,9 & 0,40 & 0,61 \\
\hline Privado & 5.719 & 69,1 & $-0,93^{* *}$ & $-0,49$ \\
\hline
\end{tabular}

Fonte: Ministério da Saúde - Sistema de Informações Hospitalares do SUS (SIH/SUS)

*Excluídos da análise os acidentes de trajeto, os outros acidentes de trabalho e as outras causas externas com percentual de $6,8 \%$ do total de dados.

${ }^{* *}$ Correlação significante com $p<0,05$. 


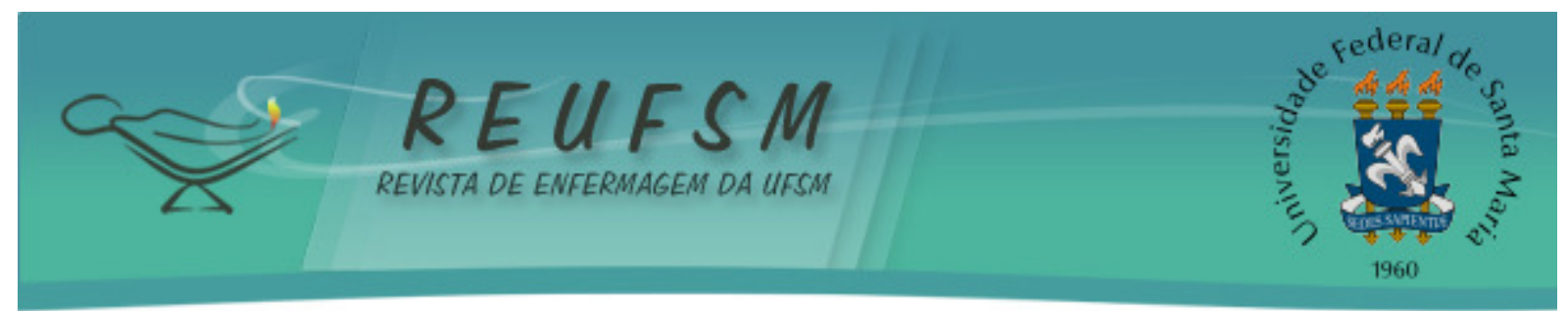

Um estudo ${ }^{12}$, publicado em 2012, avaliou as diferenças de atendimento a vítimas de acidentes e violências entre o setor público e privado. Os dados do estudo indicaram que em 2008 69,6\% das hospitalizações do país ocorreram em serviços públicos ou contratados ao SUS. Outro fator relevante, diz respeito a maior gravidade dos atendimentos realizados pelo setor público, que atende a maioria dos casos de traumatismo e politraumatismo, enquanto o setor privado é responsável pelos atendimentos de menor gravidade. ${ }^{12}$

A respeito das internações hospitalares por causas externas, estimativas indicam um crescimento de $19,1 \%$ nas taxas ao longo da última década, números que podem, em parte, ajudar a explicar os achados da Tabela 3, no que diz respeito às elevadas taxas de mortalidade e permanência hospitalar do setor público em relação ao setor privado. ${ }^{10,12}$

No que se refere, especificamente, as quedas, o setor público foi responsável por $67,1 \%$ dos atendimentos em serviços de urgência e emergência e o local de ocorrência mais registrado foi o domicílio para idosos e crianças de 10 anos ou menos. Salienta-se que, em relação as quedas, a procura por serviços públicos pode estar relacionada a especialidade do serviço, a presença de unidade de terapia intensiva e a habilidade previamente reconhecida do corpo clínico. ${ }^{12}$

A figura 2 mostra a distribuição da taxa de mortalidade oriunda das internações por quedas em hospitais segundo as macrorregiões de saúde do RS.

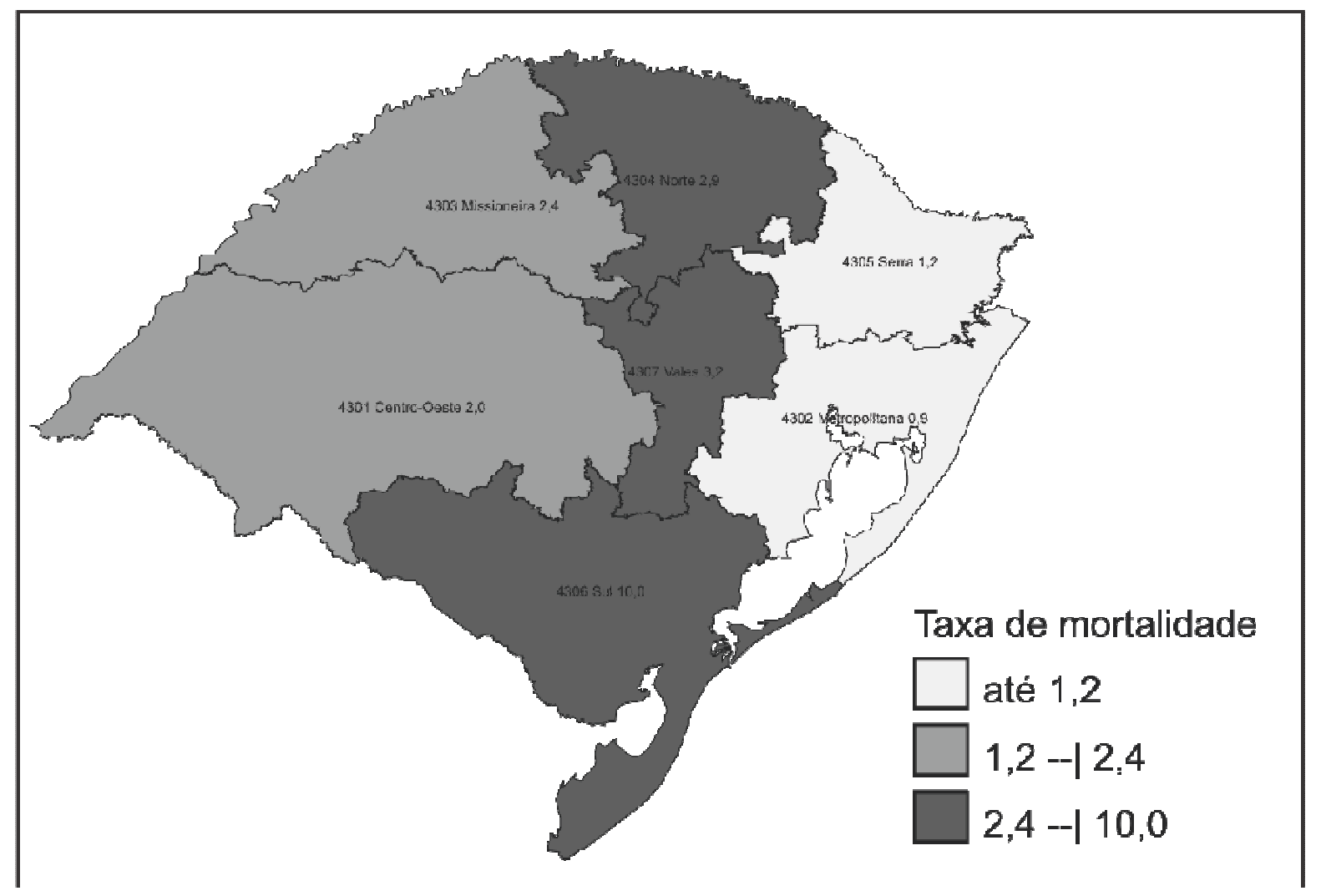

Fonte: Ministério da Saúde - Sistema de Informações Hospitalares do SUS (SIH/SUS)

Categorias CID-10: W01 - Queda no mesmo nível por escorregão, tropeção ou passos em falsos, W06 - Queda de um leito, W07 - Queda de uma cadeira, W08 - Queda de outro tipo de mobília e W10 Queda em ou de escadas ou degraus.

Figura 2 - Taxa de mortalidade das internações por quedas no período de 2008 a 2012, no Rio Grande do Sul. 


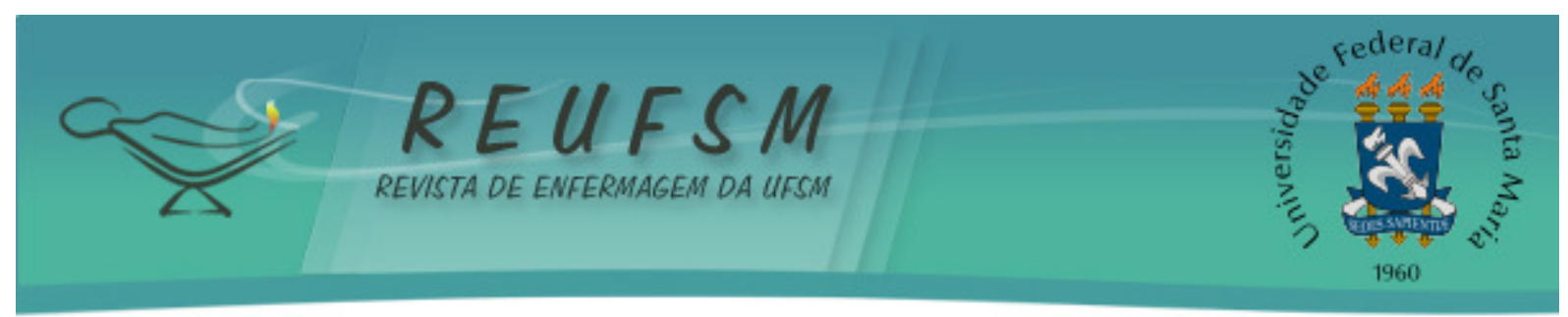

A maior incidência foi identificada nas regiões sul, central e noroeste do estado do RS. Essa distribuição espacial sugere que a ocorrência de uma mortalidade aumentada nessas regiões do Estado pode estar relacionada ao acesso e a capacidade de resolutividade dos serviços de saúde locais, pois a dificuldade em acessar serviços de maior complexidade com rapidez pode levar ao agravamento dos casos, com piora do prognóstico. ${ }^{11}$ Outra perspectiva de análise pode estar relacionada ao tipo de agravo decorrente da queda e sua gravidade, como fratura ou coma, demonstrando que esses eventos podem aumentar a incidência de mortalidade.

A taxa de mortalidade das internações por quedas, também pode sugerir a influência de fatores sociais e econômicos no desencadeamento das quedas. A adequação do ambiente bem como a possibilidade de um conjunto de ações e mudanças estruturais se faz, a partir da readequação do domicílio. Nesse sentido, reconhece-se que regiões economicamente mais desfavorecidas e com piores condições de vida e desenvolvimento, possam justificar, em termos territoriais, as altas taxas de mortalidade hospitalar encontradas nos estudos. ${ }^{13-14}$

\section{CONSIDERAÇOES FINAIS}

Os resultados encontrados são fruto de uma abordagem exploratória dos dados do Ministério da Saúde (MS), não sendo possível, portanto, serem utilizados como afirmações categóricas sem a sua devida contextualização. Sugere-se como principal contribuição ao estudo das quedas no RS, algumas inferências que podem ser utilizadas pelos órgãos governamentais de gestão da saúde para o melhor conhecimento do comportamento epidemiológico do evento no estado, a fim de subsidiar políticas públicas preventivas e protetoras da vida e bem estar social. Nesse sentido, aponta-se que para uma redução das taxas de hospitalização nos últimos anos e que as internações por quedas do mesmo nível com origem em escorregões, tropeção ou passos em falso são as principais causas do agravo investigado. Esses fatores predisponentes podem ser evitados por meio de ações preventivas, educativas em saúde e adequação dos ambientes domiciliares.

0 reconhecimento dos idosos como uma população de indivíduos mais vulneráveis as sofrerem esse evento, possibilita a Enfermagem, enquanto ação prática e educativa do cuidado planeja-lo com vistas a melhorar a capacidade funcional e a autonomia dos idosos, para manter suas atividades rotineiras em uma sociedade em constante evolução. Para isso, é necessário reconhecer a influência dos fatores ambientais em relação às quedas, traçar medidas preventivas adaptadas as condições físicas, mentais e socioeconômicas dos indivíduos, reduzindo as taxas de hospitalização e de mortalidade hospitalar por essas causas.

As taxas de mortalidade hospitalar e o regime de atendimento aos casos de internação por quedas no RS evidencia a necessidade de reestruturação do acesso (oferta) e da acessibilidade para serviços de saúde dentro das macrorregiões. Os dados sugerem que os serviços públicos absorvem os casos de maior gravidade, o que evidencia a necessidade de rearranjo entre oferta e procura desses serviços ao se tomar por base as taxas de mortalidade apresentadas. Aos leitos contratados pelo SUS, cabem os casos de menor gravidade, o que pode servir de indicador para as readequações necessárias a redução dessas taxas.

A implementação de medidas ambientais de proteção contra quedas necessita pautar-se na melhoria da qualidade de vida individual e familiar. Entende-se que, especialmente os enfermeiros de família, podem impactar na redução desses eventos com base no acompanhamento do cotidiano familiar. Desta forma, medidas preventivas e educativas adotadas, em um curto espaço de tempo, contribuem para a melhoria da estrutura e da vida de indivíduos e coletividade, prevenindo sofrimento de vítimas e 


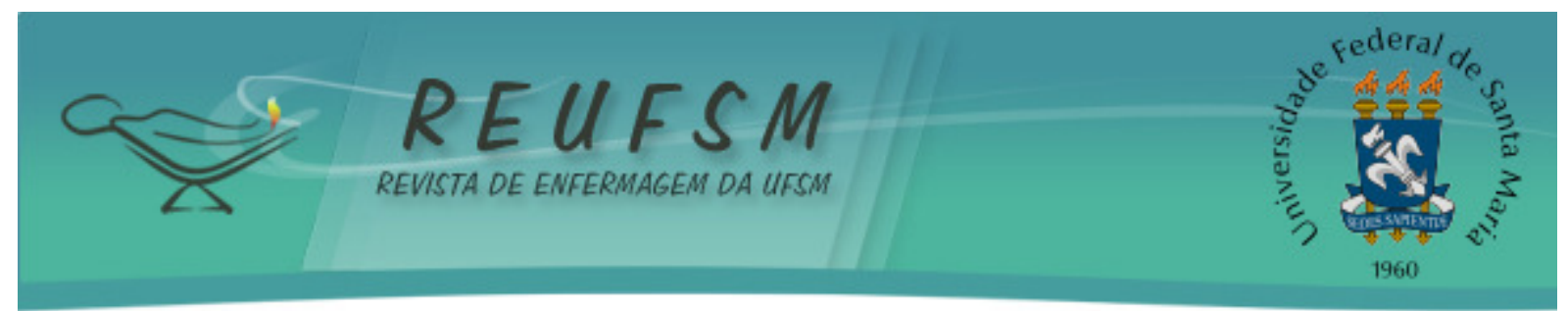

familiares, além de diminuir o número de internações e, consequentemente, os gastos públicos com tratamento e reabilitação.

Além das intervençoes educativas, os profissionais da enfermagem, poderiam atuar na redução das barreiras ambientais que contribuem para as pessoas cairem, por exemplo: redução de tapetes e degraus, adoção de material emborrachado no piso, barras de apoio para suporte no banheiro e em locais de circulação, camas com guardas de proteção, iluminação interna dos comodos sensivel ao movimento, entre outros. Algumas perspectivas de estudos para esse campo, poderiam ser pesquisas de intervenção no contexto domiciliar, a fim de testar a redução de quedas quando um enfermeiro aplica medidas e orientações preventivas no controle dos fatores de riscos ambientais identificados como promotores destes eventos.

Por fim, o desenvolvimento de práticas educativas e preventivas é capaz de impactar positivamente na redução das taxas referente as quedas no RS. 0 estudo de práticas e propostas de ação, nesse sentido, encontra-se como campo aberto para ser explorado e discutido no âmbito da academia, das práticas profissionais, da gestão e serviços de saúde. Como desafio prioritário para essa temática, ressalta-se a necessidade de investimentos em qualificação e contingente de profissionais atuando em serviços que possa dedicar tempo em visitas domiciliares para promoção da saúde, intervenções preventivas e rearranjos familiares e estruturais capazes de diminuir o risco de queda e melhorar a qualidade de vida de pessoas e coletividades.

\section{REFERÊNCIAS}

1. Brasil. Ministério da Saúde. Anexo I: Protocolo prevenção de quedas [Internet]. Ministério da Saúde/Anvisa/Fiocruz; 2013 [acesso em 2013 nov 28]. Disponível em: http://portalsaude.saude.gov.br/portalsaude/arquivos/pdf/2013/Mai/06/protocolos_CP_n 6_2013.pdf.

2. Robertson MC, Devlin N, Gardner MM, Campbell. Effectiveness and economic evaluation of a nurse delivered home exercise programme to prevent falls. RandomisedcontrolledTrial. BMJ. 2001;(322):1-6.

3. Brasil. Ministério da Saúde. Datasus. Informações de Saúde. Epidemiológicas e morbidade [Internet]. [acesso em 2013 nov 23]. Disponível em: http://www2.datasus.gov.br/DATASUS/index.php?area=0203.

4. Brasil. Ministério da Saúde. Datasus. Internações Hospitalares do SUS por local de internação: notas técnicas [Internet]. [acesso em 2013 nov 19]. Disponível em: http: / /tabnet.datasus.gov.br/cgi/sih/rxdescr.htm.

5. Nicolussi AC, Fhon TRS, Santos CAV, Kusumota L, Marques S, Rodrigues RAP. Qualidade de vida em idosos que sofreram quedas: revisão integrativa da literatura. Ciênc Saúde Coletiva. 2012;17(3):723-30.

6. Paiva MCMS, Paiva SAR, Berti HW, Campana AS. Caracterização das quedas de pacientes segundo a notificação de boletins de eventos adversos. Rev Esc Enferm USP. 2010;44(1):134-8.

7. Santos SSC, Silva ME, Pinho LB, Gautério DP, Pelzer MT, Silveira RS. Risco de quedas em idosos: revisão integrativa pelo diagnóstico da North American Nursing Diagnosis Association. Rev Esc Enferm USP. 2012;46(5):1227-36.

8. Rouquayrol MZ, Ameida Filho N. Epidemiologia e saúde. $5^{\mathrm{a}}$ ed. Rio de Janeiro: MEDSI; 2003. 


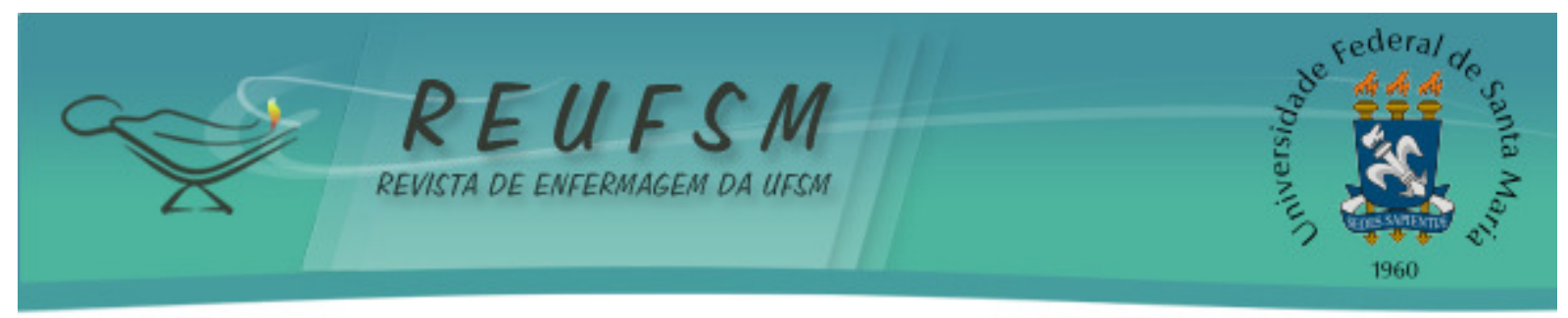

9. Siqueira FV, Facchini LA, Piccini RX, Tomasi E, Thumé E, Silveira DS, et al. Prevalência de quedas em idosos e fatores associados. Rev Saúde Pública 2007;41(5):749-56.

10. Gawryszewski VP, Scarpelini S, Dib JA, Jorge MHPM, Pereira Junior GA, Morita M. Atendimentos de emergência por lesões decorrentes de causas externas: características das vítimas e local de ocorrência, Estado de São Paulo, Brasil, 2005. Cad Saude Publica. 2008;24(5):1121-9.

11. Mathias TAF, Jorge MHPM, Andrade OH. Morbimortalidade por causas externas na população idosa residente em município da região sul do Brasil. Rev Latinoam Enferm. 2006 jan-fev;14(1):17-24.

12. Belon AP, Silveiral NYJ, Barros MBA, Baldo C, Silva MMA. Atendimentos de emergência a vítimas de violências e acidentes: diferenças no perfil epidemiológico entre o setor público e o privado. VIVA - Campinas/SP, 2009. Ciênc Saúde Coletiva. 2012;17(9):2279-90.

13. Conselho Federal de Economia (Cofecon). Comissão de Desenvolvimento Regional. Mapa da distribuição espacial da renda no Brasil. $3^{\mathrm{a}}$ ed. 2011 abr.

14. Avelar JK, Pires FC, Cortes VF. Influência dos níveis de paratormônio em quedas entre idosos e adultos em hemodiálise. Rev Enferm UFSM [Internet]. 2012 [acesso 2014 maio 5];2(1):125-33. Disponível em: http://cascavel.ufsm.br/revistas/ojs2.2.2/index.php/reufsm/article/view/4547/3134.

Data de recebimento: $27 / 04 / 2014$

Data de aceite: $25 / 11 / 2014$

Contato com autor responsável: Maíra Rossetto

Endereço: Ramiro Barcellos, 1901/101, Bairro: Bom Fim, Porto Alegre, RS, Brasil. CEP: 90035-006

E-mail: maira_rossetto@hotmail.com 